\title{
Persistent Spontaneous Pneumothorax for Four Years: A Case Report
}

\section{Mizuno Y., Iwata H., Shirahashi K., Matsui M., Takemura H.}

Department of General and Cardiothoracic Surgery, Graduate School of Medicine, Gifu University, Gifu, Japan

Received February 26, 2012; Accepted October 10, 2012.

Key words: Pneumothorax - VATS lobectomy - Minimally invasive surgery Pleural decortication

\begin{abstract}
Pneumothorax, defined as the presence of air in the pleural space, is usually classified as spontaneous or traumatic; it is unusual for pneumothorax to be categorized as being acute or chronic. Even if conservative treatment is chosen, the pneumothorax is cured when air in the pleural space dissolves into the venous blood. A 50-years-old Japanese man with no prior medical history was referred to our department with a right pneumothorax and two rightsided pulmonary nodules on chest $X$-ray and $\mathrm{CT}$. The chest radiographs of past mass screening which was taken four years ago showed right pneumothorax and right-sided pulmonary nodules. From then, all chest radiograph and chest computed tomography showed right pneumothorax and pulmonary nodules. But he underwent no medical interventions. We designed to perform an operation for a treatment of right pneumothorax and the diagnosis of pulmonary tumors. We underwent right upper lobectomy and pleural decortication under video assisted thoracic surgery. We obtained pathological diagnosis of inflammatory pseudotumor and surrounding atelectasis. He was cured from pneumothorax and pulmonary tumors. A unique case of spontaneous pneumothorax presenting with a pleural air space that was confirmed by chest radiographs and computed tomography examinations over a 4-year period is reported.
\end{abstract}

Mailing Address: Yoshimasa Mizuno, MD., PhD., Department of General and Cardiothoracic Surgery, Graduate School of Medicine, Gifu University, 1-1 Yanagido, Gifu, 501-1194, Japan; Phone: +81 58230 6325; Fax: +81 58230 6326; e-mail: mizunoyoshidasa@yahoo.co.jp 


\section{Introduction}

Pneumothorax is classified into spontaneous and traumatic (Noppen and De Keukeleire, 2008). Spontaneous pneumothorax occurs without recognized lung disease (primary spontaneous pneumothorax), due to an underlying lung disease (secondary spontaneous pneumothorax), or in conjunction with menstruation (catamenial pneumothorax) (Noppen and De Keukeleire, 2008). Spontaneous pneumothorax is a disease that usually develops acutely with ipsilateral chest pain and mild dyspnea (Noppen and De Keukeleire, 2008). Because air in the pleural space dissolves into the capillary blood, if no new gas enters the pleural space, the intrapleural gas pressure tends to decrease and the lung re-expands (Cormier and Provencher, 2009). The amount of air in the pleural cavity decreases progressively, and, finally, the pleural space disappears. Therefore, in theory, pneumothorax cannot be present for a long time, and spontaneous pneumothorax is not usually classified as an acute or chronic disease. A unique case of spontaneous pneumothorax detected by chest radiographs and computed tomography (CT) examinations continuously over a 4-year period is reported.

\section{Case report}

A 50-years-old man with no prior history of cancer, infection, or any relevant occupation was referred to our department after his family doctor had found a right pneumothorax and two right-sided pulmonary nodules on chest $\mathrm{X}$-ray and CT: a 44-mm-diameter mass in the right upper lobe and a 36-mm-diameter mass in the right lower lobe. The patient had a smoking history (current smoker: 30 packs/ year), but no emphysematous change was detectable on chest CT and respiratory function testing ( $\mathrm{FEV}_{1.0} 72.1 \%$ ). He had no symptoms, and physical examination was unremarkable. There were no specific abnormalities on laboratory examination, including tumor markers or physiological testing, except for mild elevation of C-reactive protein $(1.37 \mathrm{mg} / \mathrm{dl})$. When the chest radiographs and $\mathrm{CTs}$ of past mass screening and medical examinations were examined, right pneumothorax and right-sided pulmonary nodules were detectable on the chest radiograph taken four years earlier and on the chest CT taken three years earlier. After that, all chest radiographs (8 times) and CTs (5 times) (Figure 1) showed a right pneumothorax of various degrees and right-sided pulmonary masses that grew gradually. Though he was diagnosed as having a right pneumothorax, he underwent no treatment because he had no symptoms, and the pneumothorax on chest radiographs and chest CTs was small over the 4-year period. Regarding the pulmonary nodules, though he had been recommended to undergo the detailed examinations by his family doctor, he had been rejecting. Most recently, 18F-fluorodeoxyglucose positron emission tomography (18F-FDG PET)/CT and bronchofiberscopy were performed by his family doctor. 18F-FDG PET/CT showed no areas of increased 18F-FDG uptake, including the pulmonary masses. Bronchofiberscopy revealed no malignant findings. With a diagnosis of right pneumothorax and pulmonary tumors, 


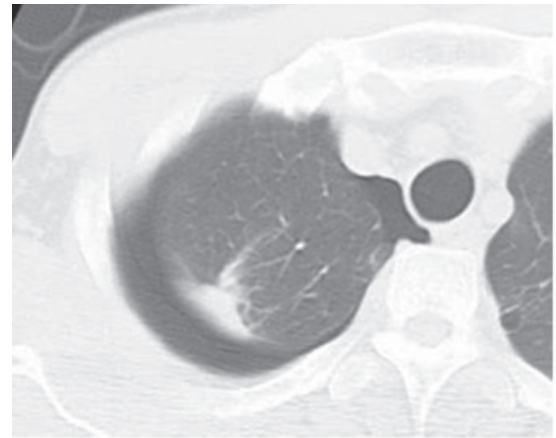

2007.11.20

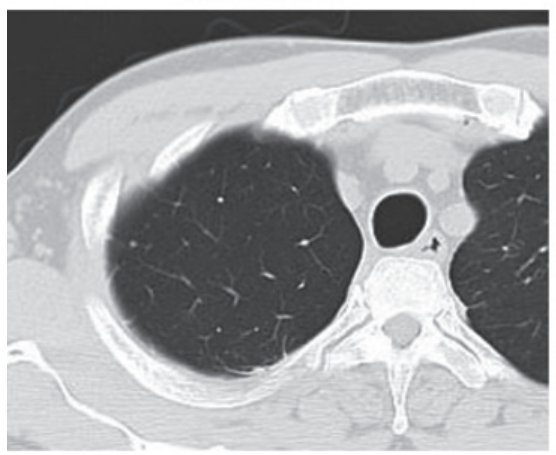

Post-operation

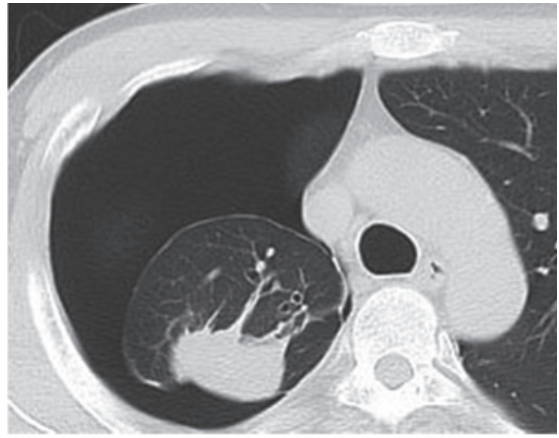

2010.9.16

Figure 1 - The findings of chest computed tomography (CT). Chest CTs before pulmonary resection show pneumothorax with a pulmonary nodule in the right upper lobe. The chest CT after surgery shows no pleural air space.
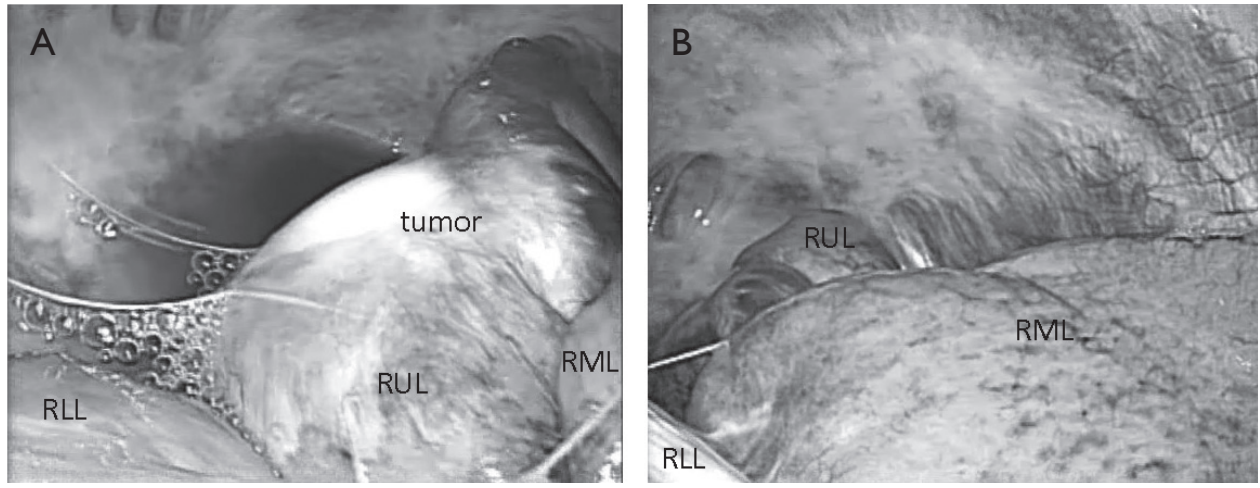

Figure 2 - The intraoperative findings.

A:The pulmonary nodule located in the right upper lobe (RUL) is white in colour. Inflammatory change and moderate thickness of the visceral pleura on the periphery of the white pulmonary nodule are detectable. A serous pleural effusion is present.

$B:$ There is little inflammatory reaction in the visceral pleura of the right middle lobe (RML) and the right lower lobe (RLL). There are scattered, moderate inflammatory lesions of the parietal pleura, and many of them are seen in the area of the pulmonary apex. 
an operation to treat the pneumothorax and obtain a pathological diagnosis of the pulmonary tumors was performed.

The intrathoracic findings are shown in Figure 2. There were no intrathoracic adhesions. The visceral pleura of the right upper lobe had inflammatory changes, and white masses in the right upper and lower lobes were easily detectable. The right upper lobe was collapsed and unexpandable, even under positive airway pressure. The visceral pleura of the right middle lobe and the right lower lobe except for the white lesion and its periphery had little inflammatory change. Therefore, the lobes were expandable easily under positive airway pressure. There was a small serous pleural effusion, and fluid was collected for general and acid-fast bacterial cultures. Moderate inflammatory lesions of the parietal pleura were scattered in the area of the pulmonary apex. A right upper lobectomy was performed by video-assisted thoracoscopic surgery (VATS). The intraoperative pathological diagnosis of the resected specimen showed no malignancy. The white mass consisted of a hypocellular fibrotic lesion, the peripheral region of which was composed of inflammatory thickening of the visceral pleura and collapsed alveoli. The patient was diagnosed as having an inflammatory pseudotumor and surrounding atelectasis. Since the right lower lobe mass appeared to be
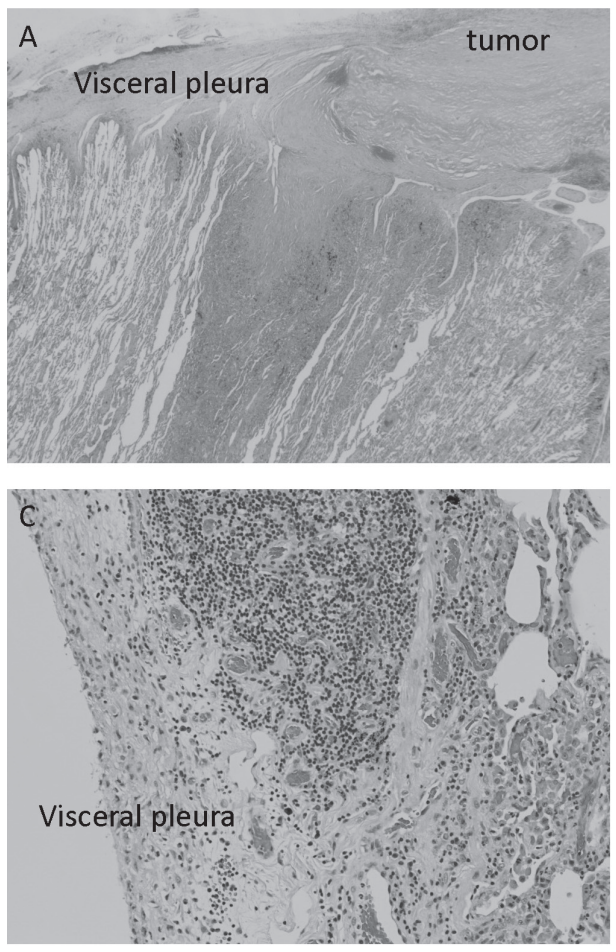

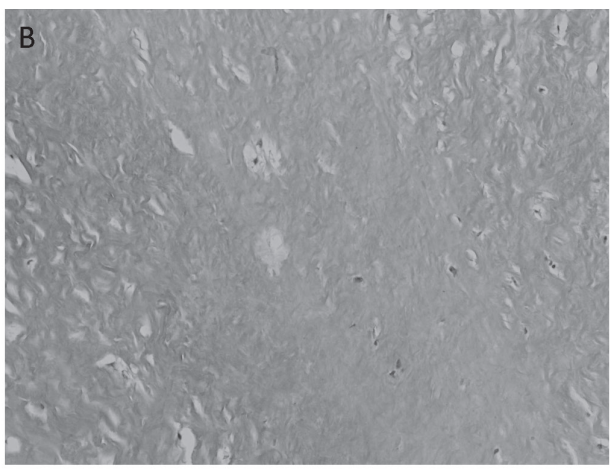

Figure 3 - The pathological findings.

A:The mass is a hypocellular fibrotic lesion, and the peripheral visceral pleura of the mass also show hypocellular fibrotic change.

$B:$ There are few cells in the tumor on high-power examination.

$C:$ The visceral pleura of the right upper lobe (RUL), which is far from the tumor, shows mild inflammatory change and thickening. 
the same lesion, pleural decortication was performed, along with biopsy of the parietal pleura. The patient's clinical course has been uneventful. The bacterial cultures showed no growth. The final pathological diagnosis was inflammatory pseudotumors with surrounding atelectasis and pleuritis (Figure 3). Chest CT 2 months after the operation showed no residual pleural cavity (Figure 1). The pulmonary nodule in the right lower lobe gradually decreased in size on the chest radiographs.

\section{Discussion}

Spontaneous pneumothorax is classified into primary spontaneous pneumothorax and secondary spontaneous pneumothorax (Noppen and De Keukeleire, 2008; MacDuff et al., 2010). Flowcharts for managing spontaneous pneumothorax have been described for both primary and secondary cases (Noppen and De Keukeleire, 2008; MacDuff et al., 2010). Many therapeutic options are available for treating spontaneous pneumothorax. If patients are not dyspneic and have a small pneumothorax on chest radiographs, observation is a therapeutic option (Noppen and De Keukeleire, 2008; MacDuff et al., 2010). Since gases are resorbed into the venous blood and no new gases enter the pleural space, the amount of air in the pleural cavity decreases progressively and the lung re-expands (Cormier and Provencher, 2009). It has been reported that the average rate of lung re-expansion with conservative treatment for spontaneous pneumothorax was 1.25 to $2.2 \%$ per day (Kircher and Swartzel, 1954; Kelly et al., 2006). Therefore, it is unusual for pneumothorax to persist continuously for a long time. Conversely, unexpandable lung is reported to be a mechanical complication resulting in the inability of the lung to expand to the chest wall (Huggins et al., 2010). Huggins et al. (2010) proposed that unexpandable lung be divided into trapped lung and lung entrapment. Trapped lung and lung entrapment were defined as the sequels of remote pleural space inflammation resulting in the development of a mature, fibrous membrane that impedes lung expansion during fluid removal and a complication of active pleural inflammation, malignancy, or hemothorax, respectively (Huggins et al., 2010). Since the present case did not have obvious past history of inflammatory disease of the lung or pleura, it did not strictly fulfil the definition of unexpandable lung proposed by Huggins et al. (2010). However, the operative and histological findings showed pleural inflammation, it may be appropriate that the present case was a subtype of trapped lung. We hypothesized that the pneumothorax was caused by a pleural laceration due to hyperstraining of the visceral pleura when the lung re-expanded, and the lung collapse and inflammatory reaction induced closure and healing of the pleural wound. We considered that the repetition of these processes caused the condition of persistent pneumothorax. This case was very unique that all clinical process developed spontaneously and fulfilled the criteria as follows: (1) continuously detectable pneumothorax on all chest radiographs and CTs; (2) no obvious past history of pleural diseases; 
(3) no past history of thoracic trauma or medical intervention that could cause pneumothorax; and (4) gas-producing bacteria were not detected on pleural effusion cultures. Because of small pleural air space, he received conservative treatment. The inflammatory pseudotumor increased in the clinical course, it was necessary for him to undergo right upper lobectomy.

\section{Conclusion}

A surgical intervention should be performed for diagnosis and treatment when persistent pneumothorax and mass lesions existed even if patients had small pleural air space and fulfilled the above-mentioned criteria.

\section{References}

Cormier, Y., Provencher, S. (2009) Resorption of gases from the pleural space. In: General Thoracic Surgery, $7^{\text {th }}$ Ed., eds. Shields, T. W., LoCicero, J., Reed, C. E., Feins, R. H., pp. 735-737, Lippincott Williams and Wilkins, Philadelphia.

Huggins, J. T., Doelken, P., Sahn, S. A. (2010) The unexpandable lung. FI000 Medicine Reports 2, 77.

Kelly, A. M., Loy, J., Tsang, A. Y., Graham, C. A. (2006) Estimating the rate of re-expansion of spontaneous pneumothorax by a formula derived from computed tomography volumetry studies. Emerg. Med. J. 23, 780-782.

Kircher, L. T. Jr., Swartzel, R. L. (1954) Spontaneous pneumothorax and its treatment. JAMA 155, $24-29$.

MacDuff, A., Arnold, A., Harvey, J.; BTS Pleural Disease Guideline Group (2010) Management of spontaneous pneumothorax: British Thoracic Society Pleural Disease Guideline 2010. Thorax 65, 18-31 (Suppl. 2).

Noppen, M., De Keukeleire, T. (2008) Pneumothorax. Respiration 76, 121-127. 